\title{
Lift and Thrust Characteristics of Flapping Wing Aerial Vehicle with Pitching and Flapping Motion
}

\author{
Chunjin Yu',2, Daewon Kim², Yi Zhao' \\ ${ }^{1}$ Institute of Flight Vehicle Engineering, Nanchang Hangkong University, Nanchang, China \\ ${ }^{2}$ Department of Aerospace Engineering, Embry-Riddle Aeronautical University, Daytona Beach, USA \\ Email: 415765032@qq.com
}

Received August 2014

\begin{abstract}
Development of flapping wing aerial vehicle (FWAV) has been of interest in the aerospace community with ongoing research into unsteady and low Reynolds number aerodynamics based on the vortex lattice method. Most of the previous research has been about pitching and plunging motion of the FWAV. With pitching and flapping motion of FMAV, people usually study it by experiment, and little work has been done by numerical calculation. In this paper, three-dimension unsteady vortex lattice method is applied to study the lift and thrust of FWAV with pitching and flapping motion. The results show that: 1) Lift is mainly produced during down stroke, however, thrust is produced during both down stroke and upstroke. The lift and thrust produced during down stroke are much more than that produced during upstroke. 2) Lift and thrust increase with the increase of flapping frequency; 3) Thrust increases with the increase of flapping amplitude, but the lift decreases with the increase of flapping amplitude; 4) Lift and thrust increase with the increase of mean pitching angle, but the effect on lift is much more than on thrust. This research is helpful to understand the flight mechanism of birds, thus improving the design of FWAV simulating birds.
\end{abstract}

\section{Keywords}

Flapping Wing, Aerial Vehicle, Lift Characteristics, Thrust Characteristics

\section{Introduction}

All flying animals in the nature use flapping-wing flight mode. For more than a decade, flapping wing aerial vehicle (FWAV) inspired by biological flyers such as insects and birds are suitable for missions involving confined spaces, such as buildings or short distances. Therefore, studying flapping-wing aerial vehicles have a wide application foreground.

Recent studies have uncovered previously unknown unsteady aerodynamic mechanisms of flapping flight. These include the clap and fling [1], leading edge vortex generation [2], rotational lift [3], and wing-wing interaction [4] mechanisms, which help explain the basic principles of unsteady force generation in flight. The aerodynamics of insect wings is entirely different from the conventional wings. However Kesel [5] showed that 
the wings of dragonfly is comparable wing conventional profiles in a steady flow, but unlike conventional profiles negative pressure occurs on both the upper and lower surfaces of the wing at angles of attack from $-10^{0}$ to $0^{0}$. Reduced frequency increases with increase in unsteady effects and for an insect it will be greater than 0.75 . But in the case of birds [6] they are very low and crudely give their forward velocity, it is most often described by the vortex wing theory [7].

Most researchers studied a combined pitching and plunging motion of FWAV, such as Jones [8], Heath cote [9], Stewart [10], and so on. Their analysis was based on two-dimension aerodynamic model.

To research the pitching and flapping motion of flapping wing, since the complexity of airflow, people adopted three-dimension aerodynamic model to calculate the aerodynamic performance of flapping wing. Smith [11] used an unsteady aerodynamic panel method to simulate a tethered moth's flapping wings, Fitzgerald et al. [12] studied the fluid-structure interaction of flexible flapping wing systems by unsteady vortex lattice method (UVLM), and found that UVLM is suitable to calculate the unsteady aerodynamic force. Yu et al. [13] derived a formula to calculate the aerodynamic force based on three dimension unsteady vortex lattice method and vector analysis.

Some researchers carried out experimental investigation on aerodynamic performance of FWAV. Mazaheri et al. [14] designed a flapping-wing system and an experimental set-up to measure the unsteady aerodynamic forces of the flapping wing motion. Muniappan et al. [15] discussed the effect of aspect ratio and the wing planform shape on lift and thrust force experimentally.

Most work mentioned above were either on the numerical analysis or on the experimental investigation. In this paper, both numerical analysis and experimental investigation will be used to analyze the lift and thrust characteristics of FWAV with pitching and flapping motion. As an example, birds will be used to explain these reasons. This research is helpful to improve the design of FWAV.

\section{Aerodynamics-Unsteady Vortex Lattice Method}

Three-dimension unsteady vortex lattice method (UVLM) is used to calculate the aerodynamics in this paper. The diagrammatic drawing of UVLM is shown as Figure 1 by discretizing the continuous sheet on a wing into a set of ring vortices of unknown magnitude. The rings are made of four finite line segments of equal vorticity magnitude. The Kutta condition is composed on the trailing edge of the wing and no-penetration boundary condition is imposed at a set of control points. At each time step in the analysis the voracity along the trailing edge is shed into the wake. The finite segments of bound vorticity and wake vorticity induce a downwash on the wing according to the Biot-Savart law. The Biot-Savart law is then put into matrix form to find the total downwash at each control point from all of the bound ring vortices and wake vortices.

The pressures and loads can be calculated by using the unsteady Bernoulli equation:

$$
\frac{P_{r e f}-p}{\rho_{f}}=\frac{Q^{2}}{2}-\frac{v_{r e f}^{2}}{2}+\frac{\partial \Phi}{\partial t}
$$

where $P_{\text {ref }}$ is reference pressure, $p$ is pressure of fluid, $\rho_{f}$ is fluid density, $Q$ is fluid velocity, $v_{\text {ref }}$ is reference velocity, $\Phi$ is the potential of flow, $t$ is the time.

The pressure difference between the flapping-wing upper and lower surfaces is then

$$
\Delta P=P_{l}-P_{u}=\rho\left[\left(\frac{Q_{t}^{2}}{2}\right)_{u}-\left(\frac{Q_{t}^{2}}{2}\right)_{l}+\left(\frac{\partial \Phi}{\partial t}\right)_{u}-\left(\frac{\partial \Phi}{\partial t}\right)_{l}\right]
$$

where $P_{l}$ is the pressure of lower surfaces, $P_{u}$ is the pressure of upper surfaces.

The tangential velocity due to the wing vortices will have two components on the wing, and it can be approximated by the two directions, $i, j$, on the surface. The velocity of $i$ direction due to wing vortices on each element is

$$
\begin{aligned}
& \pm \frac{\partial \Phi}{\partial \tau_{i}}= \pm \frac{\Gamma_{i j}-\Gamma_{i-1, j}}{2 \Delta c_{i j}} \\
& \pm \frac{\partial \Phi}{\partial \tau_{j}}= \pm \frac{\Gamma_{i j}-\Gamma_{i, j-1}}{2 \Delta b_{i j}}
\end{aligned}
$$

where " \pm " represents the upper and lower surfaces, respectively; $\Delta c_{i j}$ and $\Delta b_{i j}$ are the panel lengths in the $i$-th and $j$-th directions. Similarly, $\tau_{i}$ and $\tau_{j}$ are the panel tangential vectors in the $i$ and $j$ directions, $\Gamma$ 


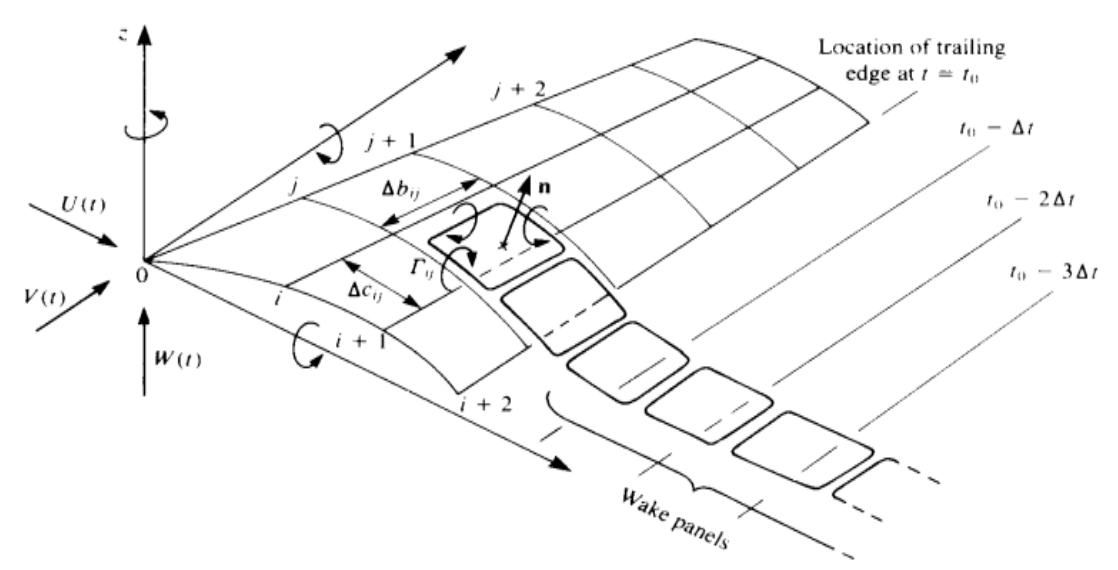

Figure 1. Diagrammatic drawing of vortex lattice method [16].

is the strength of the vortex.

Since for this vortex ring model $\Delta \Phi=\Gamma$,

$$
\pm \frac{\partial \Phi_{i j}}{\partial t}= \pm \frac{\partial}{\partial t} \frac{\Gamma_{i j}}{2}
$$

Substituting these terms into Equation (2) results in

$$
\begin{aligned}
\Delta P_{i j}= & \rho\left\{\left[(t)+u_{W}, V(t)+v_{W}, W(t)+w_{W}\right] \cdot \tau_{i} \cdot \frac{\Gamma_{i j}-\Gamma_{i-1, j}}{\Delta c_{i j}}\right. \\
& \left.+\left[U(t)+u_{W}, V(t)+v_{W}, W(t)+w_{W}\right]_{i j} \cdot \tau_{i j} \cdot \frac{\Gamma_{i j}-\Gamma_{i, j-1}}{\Delta b_{i j}}+\frac{\partial}{\partial t} \Gamma_{i j}\right\} .
\end{aligned}
$$

where $U(t), V(t), W(t)$ are respectively the fluid velocities of $X$ direction, $Y$ direction and $Z$ direction of inertial coordinate when time is $t$ moment, and $u_{W}, v_{W}, w_{W}$ are respectively the velocity of $x$ direction, $y$ direction and $z$ direction of body coordinate.

The contribution of this panel to the loads is

$$
\Delta F_{i j}=-(\Delta P \cdot \Delta S)_{i j}
$$

where $\Delta P$ is pressure of element, $\Delta S$ is area of element.

The lift can be derived based on vector transfer [13],

$$
\Delta L_{i j}=\Delta F_{i j} \cdot \cos \alpha_{i j} \cos \beta_{i j}
$$

The drag is the force component parallel to the flight direction and each panel contribution is

$$
\Delta D_{i j}=\sum \rho\left[\left(w_{\text {ind }}+w_{W}\right)_{i j}\left(\Gamma_{i j}-\Gamma_{i-1, j}\right) \Delta b_{i j}+\frac{\partial}{\partial t} \Gamma_{i j} \Delta S_{i j} \sin \alpha_{i j}\right]
$$

where $w_{\text {ind }}$ is induced velocity of flapping wing.

If the panel is at the leading edge then

$$
\Delta D_{i j}=\sum \rho\left[\left(w_{\text {ind }}+w_{W}\right)_{i j} \Gamma_{i j} \Delta b_{i j}+\frac{\partial}{\partial t} \Gamma_{i j} \Delta S_{i j} \sin \alpha_{i j}\right]
$$

If forward flight velocity is constant, then

$$
\Delta T_{i j}=-\Delta D_{i j}
$$

The total lift and thrust are obtained by integrating the contribution of each element.

$$
\begin{gathered}
L=\sum\left(\Delta F_{i j} \cdot \cos \alpha_{i j} \cos \beta_{i j}\right) \\
T=\sum \Delta T_{i j}
\end{gathered}
$$




\section{Production of Lift and Thrust}

In this paper, the motion of FWAV can be decomposed as pitching $(\alpha)$ motion and flapping $(\beta)$ motion, as shown in Figure 2.

These two motion varied with the time can be written as:

$$
\begin{gathered}
\alpha(t)=\alpha_{0}+\alpha_{1} \cos (2 \pi f t) \\
\beta(t)=\beta_{0}+\beta_{1} \cos (2 \pi f t)
\end{gathered}
$$

where $\alpha_{0}$ and $\beta_{0}$ respectively represent the mean angle of attack of pitching and flapping motion, $\alpha_{1}$ and $\beta_{1}$ are defined as the amplitude of flapping and pitching motion respectively, $f$ is flapping frequency (Hz).

The sketch of lift and thrust production during upstroke and down stroke is shown in Figure 3.

A typical wing section is observed, and the aerodynamic force can be obtained by integrating the pressure of wing surface. The lift and thrust (drag) are the components of the aerodynamic force in the Y-axis and the negative $\mathrm{X}$-axis direction, respectively. Positive thrust and negative lift are produced during upstroke, positive lift and positive thrust are produced during down stroke.

\section{Numerical Calculation}

The FMAV we studied is shown in Figure 4. The shape of flapping wing is approximate a quarter of ellipse, and the equation of ellipse is

$$
\left(\frac{x}{0.08}\right)^{2}+\left(\frac{y}{0.17}\right)^{2}=1
$$

In order to calculate the lift and thrust, we assume original parameters of flapping wing motion are: $\alpha_{0}=7.5^{\circ}, \alpha_{1}=0^{\circ}, \beta_{0}=0^{\circ}, \quad \beta_{1}=42.5^{\circ}$ fluid velocity $Q=5 \mathrm{~m} / \mathrm{s}$ and $f=5.5 \mathrm{~Hz}$.

The lift curve and thrust curve can be calculated in a flapping period by using Equation (11), and as shown in Figure 5.

Figure 5 shows that lift is mainly produced during down stroke, and drag is produced during upstroke. However, thrust is produced during both down stroke and upstroke. The lift and thrust produced during down stroke are much more than that produced during upstroke. The average lift during one flapping period $\bar{L}=12.1 \mathrm{~g}$, and the average thrust in one period $\bar{T}=11.9 \mathrm{~g}$.

\subsection{Effect of Flapping Frequency on Lift and Thrust}

In order to study the effect of flapping frequency on lift and thrust, $f$ is changed to $4.3 \mathrm{~Hz}, 6.3 \mathrm{~Hz}, 7.1 \mathrm{~Hz}$ and $7.9 \mathrm{~Hz}$ respectively, and other parameters are as same as the original parameters.

Figure 6 is the lift comparison of experiment result and numerical result, and Figure $\mathbf{7}$ is the thrust comparison of experiment result and numerical result.

Figure 6 and Figure 7 show that the lift and thrust increase with the increase of flapping frequency. To fly higher and faster, birds can increase their flapping frequency, and it means birds need consume more internal energy.

\subsection{Effect of Flapping Amplitude on Lift and Thrust}

In order to study the effect of flapping amplitude on lift and thrust, $\beta_{1}$ is changed respectively to $37.5^{\circ}, 40^{\circ}$, $45^{\circ}$ and $47.5^{\circ}$, and other parameters are as same as the original parameters.

Figure 8 shows that thrust increases with the increase of flapping amplitude, but the lift decreases with the increase of flapping amplitude. Birds can increase flapping amplitude to produce more thrust to increase flight velocity, but the weight of bird changes little, so the coefficient of lift decreases.

\subsection{Effect of Mean Pitching Angle on Lift and Thrust}

In order to study the effect of mean pitching angle on lift and thrust, $\alpha_{0}$ is changed respectively to $2.5^{\circ}, 5^{\circ}, 10^{\circ}$ and $12.5^{\circ}$, and other parameters are as same as the original parameters. 


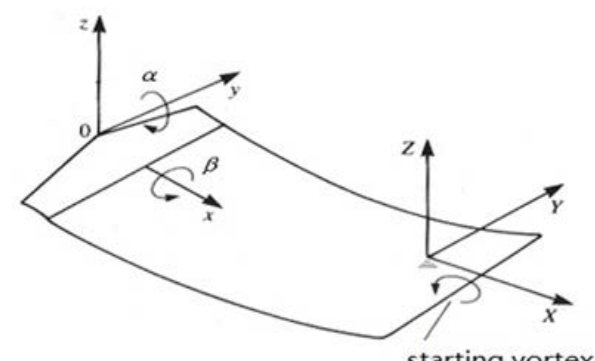

Figure 2. Motion with pitching and flapping [17].

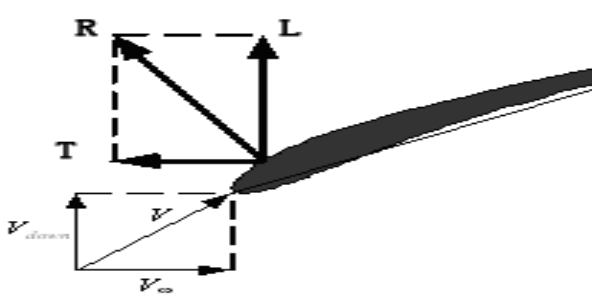

(a)

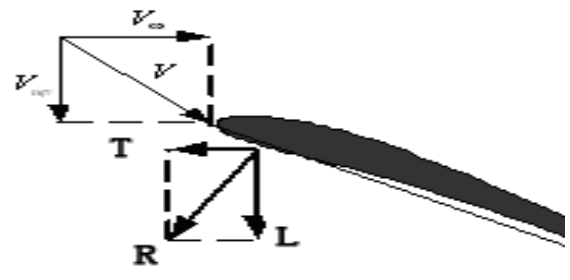

(b)

Figure 3. Sketch of lift and thrust production during upstroke and downstroke. (a) downstroke; (b) upstroke.

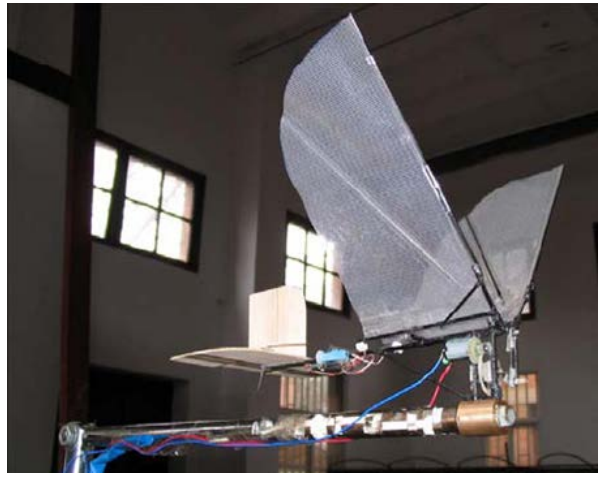

Figure 4. FMAV.

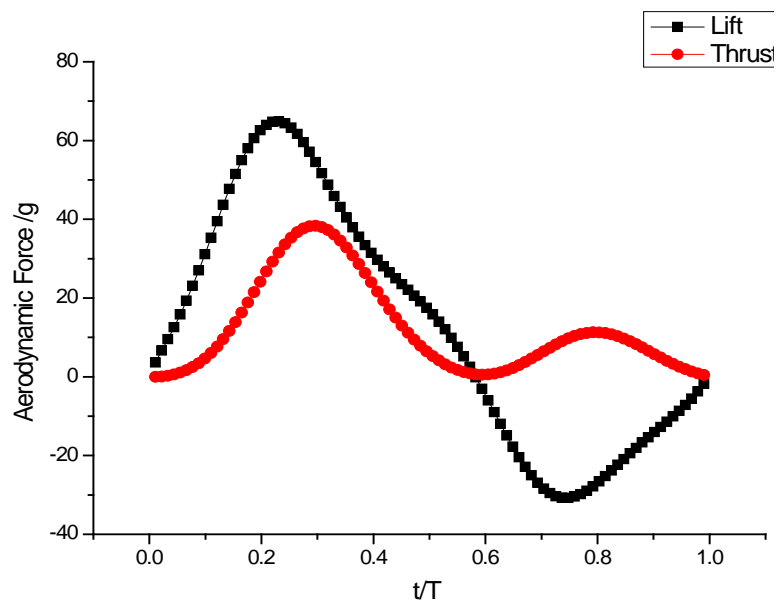

Figure 5. Lift and thrust curve in one flapping period. 


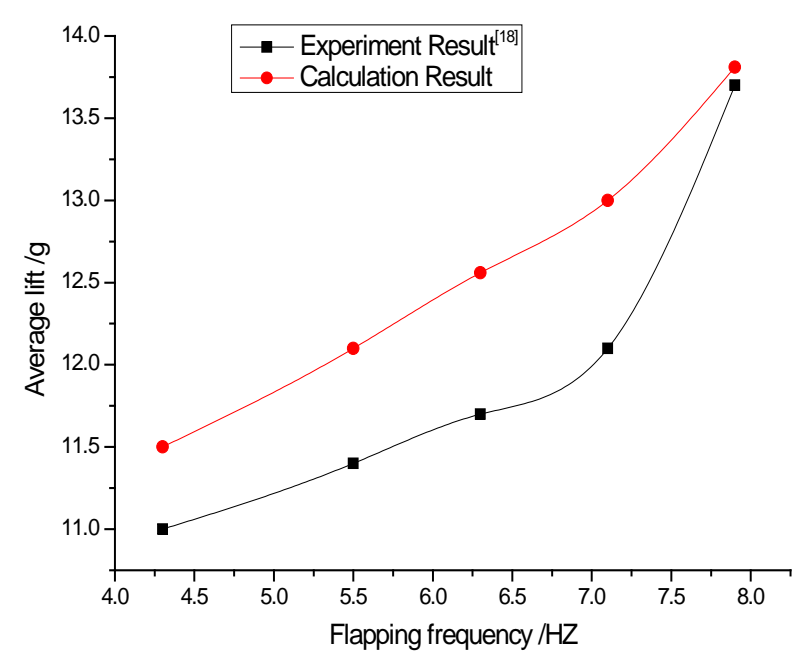

Figure 6. Effect of flapping frequency on lift.

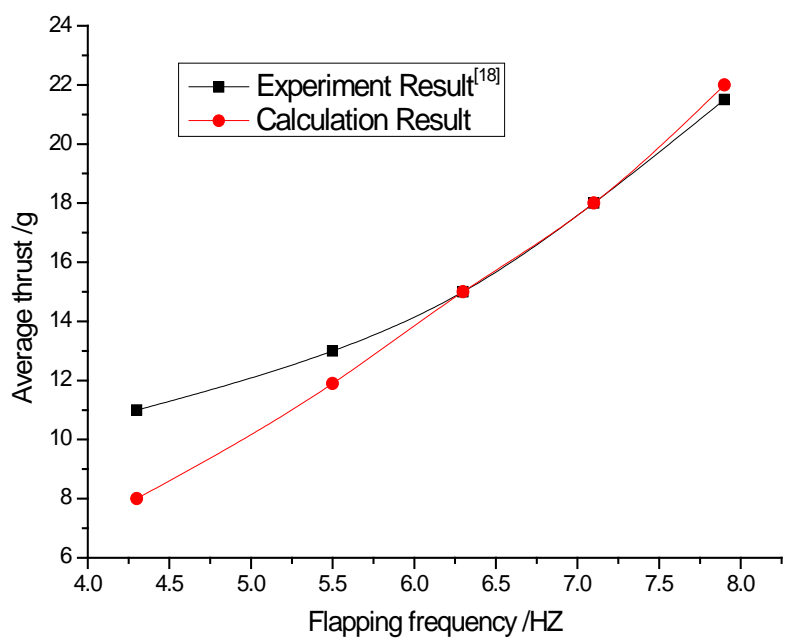

Figure 7. Effect of flapping frequency on thrust.

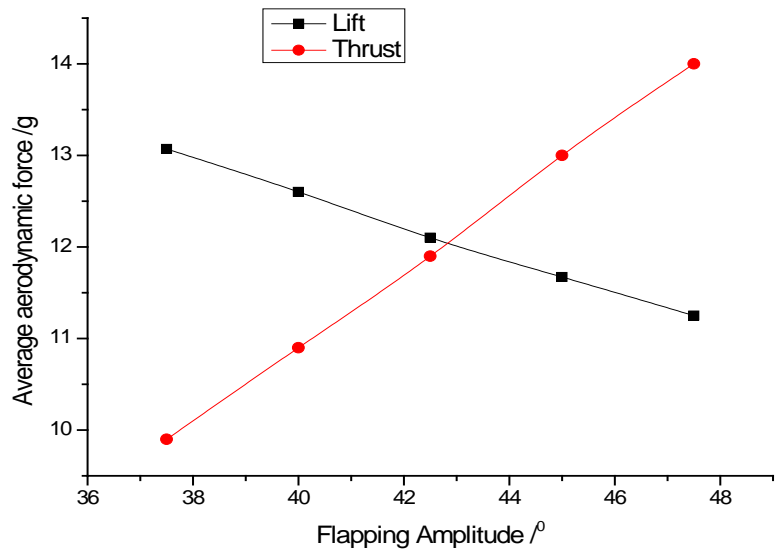

Figure 8. Effect of flapping amplitude on lift and thrust.

Figure 9 shows that the lift and thrust increase with the increase of mean pitching angle, but the effect on lift is much more than on thrust. To obtain much more lift, birds need increase mean pitching angle. 


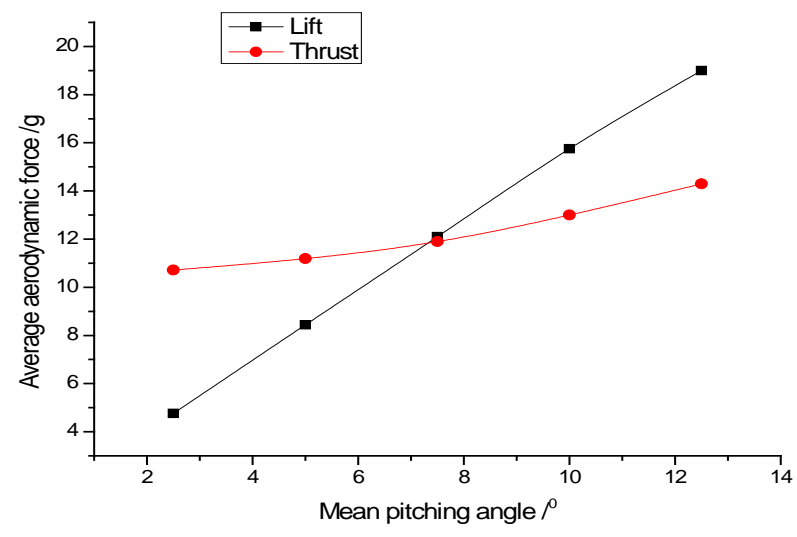

Figure 9. Effect of mean pitching angle on lift and thrust.

\section{Conclusions}

Three-dimension unsteady vortex lattice method is applied to study the lift and thrust of FWAV with pitching and flapping motion. The following conclusions can be reached based on this study:

1) Lift is mainly produced during down stroke; however, thrust is produced during both down stroke and upstroke. The lift and thrust produced during down stroke are much more than that produced during upstroke.

2) Lift and thrust increase with the increase of flapping frequency;

3) Thrust increases with the increase of flapping amplitude, but the lift decreases with the increase of flapping amplitude;

4) Lift and thrust increase with the increase of mean pitching angle, but the effect on lift is much more than on thrust.

This research is helpful to understand the flight mechanism of birds, thus improving the design of FWAV simulating birds. In the future we will continue the aerodynamic study of FWAV, such as 1) how the bound vortex and wake vortex affect the thrust in Equation (8); 2) which vortex is mainly to produce thrust; 3) effect of the section of flapping wing on aerodynamic force.

\section{Acknowledgements}

This work is financially supported by Embry-Riddle Aeronautical University, Jiangxi Provincial Department of Education (GJJ12412) and Doctorate Research Foundation of Nanchang Hang kong University (EA201006045).

\section{References}

[1] Weis-Fogh, T. (1973) Quick Estimates of Flight Fitness in Hovering Animals, including Novel Mechanisms for Lift Production. Journal of Experimental Biology, 59, 169-230.

[2] Ellington, C.P. (1984) The Aerodynamics of Hovering Flight. IV. Aerodynamic Mechanisms. Philosophical Transactions of Royal Society B, 305, 79-113. http://dx.doi.org/10.1098/rstb.1984.0052

[3] Dickinson, M.H. (1994) The Effects of Wing Rotation on Unsteady Aerodynamic Performance at Low Reynolds Numbers. Journal of Experimental Biology, 192, 179-206.

[4] Dickinson, M.H., Lehmann, F. and Sane, S.P. (1999) Wing Rotation and the Aerodynamic Basis of Insect Flight. Science, 284, 1954-1960. http://dx.doi.org/10.1126/science.284.5422.1954

[5] Kesel, A.B. (2000) Aerodynamic Characteristics of Dragonfly Wing Sections Compared with Technical Aerofoils. Journal of Experimental Biology, 203, 3125-3135.

[6] Shyy, W., Berg, M. and Ljungqvist, D. (1999) Flapping and Flexible Wings for Biological and Micro Air Vehicles. Progress in Aerospace Sciences, 35, 455-505.

[7] Obalske, B.W. and Dial, K.P. (1996) Flight Kinematics of Black-Billed Magpies and Pigeons over a Widerange of Speeds. Journal of Experimental Biology, 199, 263-280.

[8] Jones, K.D., Duggan, S.J. and Platzer, M.F. (2011) Flapping-Wing Propulsion for a Micro Air Vehicle. The 39th Aerospace Sciences Meeting \& Exhibit, Reno, 8-11 January 2001. 
[9] Heathcote, S., Wang, Z. and Gursul, I. (2008) Effect of Spanwise Flexibility on Flapping Wing Propulsion. Journal of Fluids and Structures, 24, 183-199. http://dx.doi.org/10.1016/j.jfluidstructs.2007.08.003

[10] Stewart, E.C., Patil, M.J. and Canfield, R.A. (2014) Aeroelastic Shape Optimization of a Flapping Wing. The 10th AIAA Multidisciplinary Design Optimization Conference, 13-17 January 2014, National Harbor. http://dx.doi.org/10.2514/6.2014-0469

[11] Smith, M.J.C., Wilkin, P.J. and Williams, M.H. (1996) The Advantages of an Unsteady Panel Method in Modeling the Aerodynamic Forces on a Rigid Flapping Wings. Journal of Experimental Biology, 199, 1073-1083.

[12] Fitzgerald, C., Valdez, M. and Balachandran (2011) A Comparison of Computational Models for Fluid-Structure Interaction Studies of Flexible Flapping Wing Systems. The 49th AIAA Aerospace Sciences Meeting including the New Horizons Forum and Aerospace Exposition, Orlando, 4-7 January 2011.

[13] Yu, C., Ang, H., Chen, Q., et al. (2008) Three-Dimension Unsteady Vortex Lattice Method for Flexible Structure Flapping-Wing Aerial Vehicle. Journal of Nanjing University of Aeronautics and Astronautics, 40, 451-455.

[14] Mazaheri, K. and Ebrahimi, A. (2011) Experimental Investigation on Aerodynamic Performance of a Flapping Wing Vehicle in Forward Flight. Journal of Fluids and Structures, 27, 586-595. http://dx.doi.org/10.1016/j.jfluidstructs.2011.04.001

[15] Muniappan, A. (2005) Lift and Thrust Characteristics of Flapping Wing Micro Air Vehicle(MAV). The 43rd AIAA Aerospace Sciences Meeting and Exhibit, Reno, 10-13 January 2005. http://dx.doi.org/10.2514/6.2005-1055

[16] Katz, J. and Plotkin, A. (2001) Low-Speed Aerodynamics. 2nd Edition, Cambridge University Press, New York. http://dx.doi.org/10.1017/CBO9780511810329

[17] Yu, C. (2009) Numerical Study of Aerodynamics for Flexible Membrane Flapping-Wing Aerial Vehicle. Ph.D. Thesis, Nanjing University of Aeronautics and Astronautics, Nanjing.

[18] Zeng, R. (2005) Aerodynamic Characteristics of Flapping-Wing MAV Simulating Bird Flight. Ph.D. Thesis, Nanjing University of Aeronautics and Astronautics, Nanjing. 